\title{
At the Tip of the Tongue: A Novel Human Infection by a Microsporidium
}

M. G. Metcalfe ${ }^{1}$, M. M. Choudhary ${ }^{3}$, G. S. Visvesvara ${ }^{2}$, N. J. Pieniazek ${ }^{2}$, R. D. Bandea ${ }^{2}$, C. Bern ${ }^{2}$, P. Adem $^{1}$, K. Arrambide ${ }^{3}$, M. U. Saeed ${ }^{3}$ and S. R. Zaki ${ }^{1}$

${ }^{1}$ Infectious Disease Pathology Branch, ${ }^{2}$ Division of Parasitic Diseases, CDC, Atlanta, GA 30333

${ }^{3}$ Southern Illinois University, Infectious Diseases, Quincy, IL 62301

Microsporidia are unicellular obligate intracellular parasites that infect a wide range of invertebrates and vertebrates. At least 8 microsporidian genera have been identified as human pathogens:

Anncaliia (previously Brachiola), Encephalitozoon, Enterocytozoon, Vittaforma, Nosema, Pleistophora, Trachipleistophora, and 'Microsporidium' (a collective term for microsporidia for which the genus has not been determined) [1]. The majority of human microsporidium infections have been described as causing chronic diarrhea in patients with HIV/AIDS; however, ocular infections have also been documented in healthy individuals [2].

In 2009, a 67 year old woman presented with tongue nodules. At the time of presentation, the woman was immunosurppressed due to treatment for non-Hodgkin's lymphoma. Histopathology showed myositis characterized by clusters of small, ovoid, basophilic organisms within the myocytes. The microorganisms were also noted by Giemsa, Warthin-Starry silver, and Lillie-Twort Gram stains. An onslide embedding protocol [3] was modified to deparaffinize, resin-embed and thin section the tissue.

Abundant 2-3 $\mu \mathrm{m}$ spores in the myocytes were seen by light and electron microscopy (Fig.1-6). The polar filament coiled around the sporoplasm 10-14 times (Fig.5). Additionally, the polar filament was anisofilar with the last few coils being smaller in diameter and the nuclei were in a diplokaryotic arrangement (Fig.6). Other morphological features included lamellar polaroplast, manubroid, posterior vacuole, and ribosomes.

PCR of formalin-fixed paraffin-embedded tissue resulted in the identification of a microsporidium that is closely related to Tubulinosema acridophagus. Tubulinosema acridophagus is a parasite found in North American grasshoppers such as Melanoplus and Schistocerca [4] and probably in the fruit fly Drosophila melanogaster [5]. Prior to this case, the genus Tubulinosema had not been associated with human infection.

Electron microscopy is a powerful technique in conjunction with molecular and histopathological analyses. Electron microscopy aided in the diagnosis of this case and specific PCR testing which lead to identification of a new species of microsporidium associated with human infection.

References

[1] A. Mathis et al., Clinical Microbiology Reviews 18 (2005) 423.

[2] C.M.L. Chan et al., Ophthalmology 110 (2003) 1420.

[3] M.A. Hayat, Principles and Techniques of Electron Microscopy, 3rd ed., CRC Press, Inc. Boca Raton, FL, 1989.

[4] J.E. Henry. Journal of Invertebrate Pathology 9 (1967) 331.

[5] C. Franzen et al., Journal of Eukaryotic Microbiology 52 (2005) 141. 

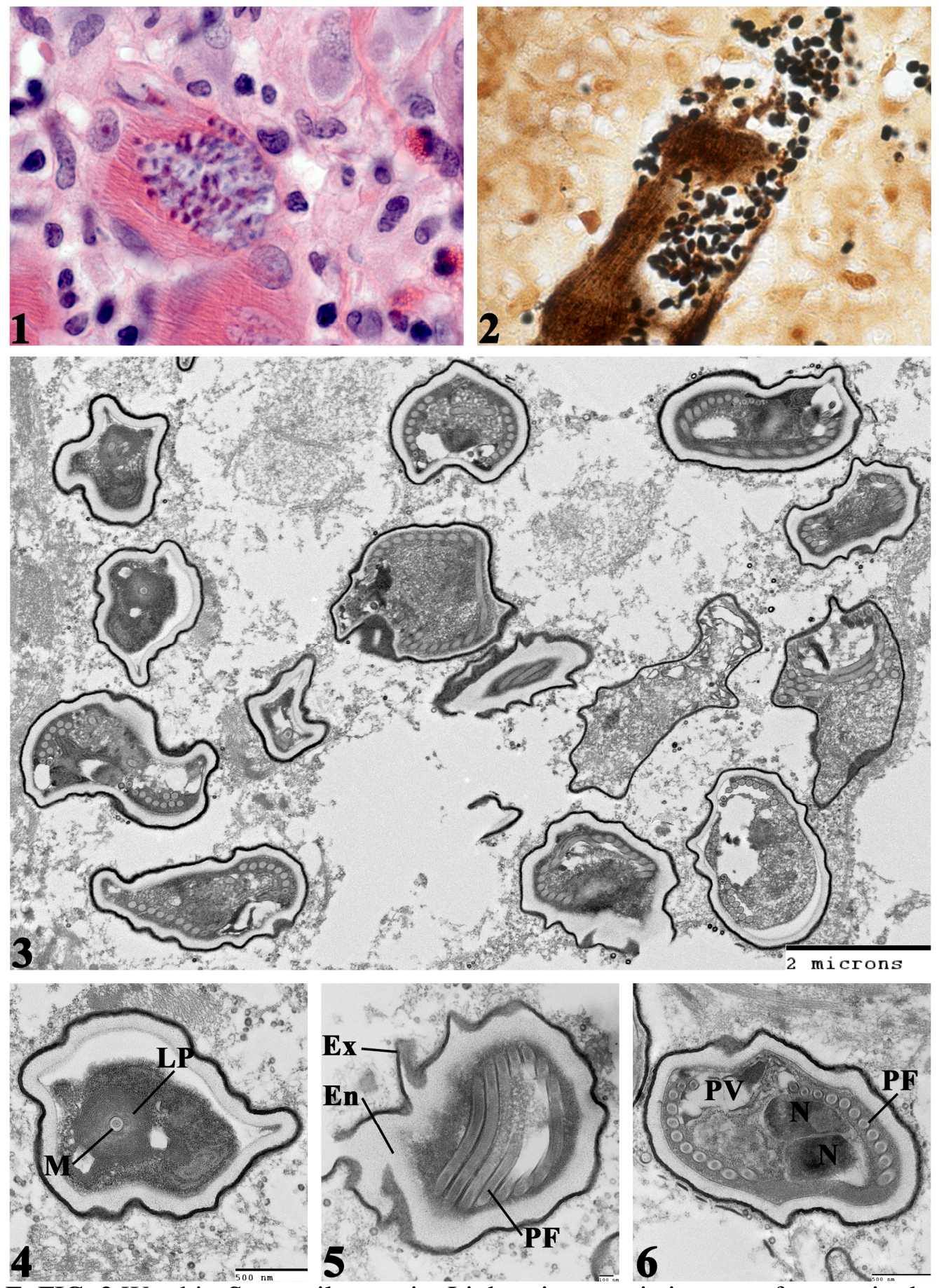

FIG. 1.H\&E. FIG. 2.Warthin-Starry silver stain. Light microscopic images of spores in the tongue myocytes (100 x objective). FIG. 3. Electron micrograph (EM) of numerous spores. FIG. 4. EM of a spore showing a cross-section of the manubroid (M), which is the straight portion of the polar filament surrounded by the lamellar polaroplast $(\mathbf{L P})$. Bar $=500 \mathrm{~nm}$. FIG. 5. An electron dense exospore (Ex) and electron lucent endospore (En) compose the spore wall. A limited number of polar filaments $(\mathbf{P F})$ are visible. Bar $=100 \mathrm{~nm}$. FIG. 6. The contents of the spore (sporoplasm) are made up of a posterior vacuole (PV), diplokaryon $(\mathbf{N})$, ribosomes, polaroplast and polar filaments (PF) which wraps around the area between the nuclei and the endospore layer. Bar $=100 \mathrm{~nm}$. 